\title{
Representation of Odor Habituation and Timing in the Hippocampus
}

\author{
Sachin S. Deshmukh and Upinder S. Bhalla \\ National Centre for Biological Sciences, Gandhi Krishi Vignana Kendra Campus, Bangalore 560065, India
}

We performed simultaneous single-neuron recordings from the hippocampus and the olfactory bulb of anesthetized, freely breathing rats. Odor response properties of neurons in the olfactory bulb and hippocampus were characterized as firing rate changes or respirationcoupled changes. A panel of five odors was used. The rats had not been exposed to the odors on the panel before the experiment. The olfactory bulb and hippocampal neurons responded to repeated odor presentations in two ways: first, by changes in firing rate, and second, by respiratory tuning changes. Approximately $60 \%$ of bulbar neurons, $48 \%$ of hippocampal CA1 neurons, and $12 \%$ of hippocampal CA3 neurons showed statistically significant responses. None of the odor-responsive neurons in either the bulb or hippocampus responded to all of the odors on the panel. Repeated $10 \mathrm{sec}$ odor stimuli presented at the intervals of 20,30,60,110, and $160 \mathrm{sec}$ were used to analyze the effect of the interval on odor response properties of the recorded neurons. Bulbar neurons were relatively nonselective for odor interval. Hippocampal neurons showed unexpected selectivity for the interval between repeated odor presentations. CA1 and CA3 neurons responded to only one to three of the intervals in the range. On the basis of these findings, we postulate that the hippocampus has the ability to keep track of the time elapsed between consecutive odor stimuli. This may act as a neuronal substrate for habituation and for complex tasks such as odor-guided navigation.

Key words: olfactory bulb; hippocampus; odor; odor selectivity; habituation; information processing; representation; electrophysiology; single-neuron recording

\section{Introduction}

Sensory habituation is thought to play a role in filtering of less significant or predictable events. Habituation to prolonged or repeated presentation of odors is a well documented phenomenon. The hippocampus (HC) has been implicated in odor learning (Eichenbaum et al., 1991) and odor habituation (Poellinger et al., 2001). We examine the role of HC in odor perception and habituation.

Hippocampal involvement in olfactory perception is well documented. Anatomically, the lateral entorhinal cortex is known to receive inputs from the olfactory bulb $(\mathrm{OB})$ and piriform cortex. The perforant pathway from the entorhinal cortex excites the dentate gyrus, CA1, and CA3, linking the olfactory sensory regions with the HC (Lynch and Granger, 1991). Field potential studies have shown rhythmical slow activity in the $\mathrm{HC}$ and the dentate gyrus in response to olfactory stimulants such as predator urine and xylene (Vanderwolf, 1992). Electrical stimulation of the OB elicits single-neuron and field potential responses in CA3 (Yokota et al., 1967; Habets et al., 1980).

Functional magnetic resonance imaging studies in humans have implicated HC along with primary olfactory cortex and anterior insula in odor habituation (Poellinger et al., 2001). HC

\footnotetext{
Received Aug. 14, 2002; revised Dec. 4, 2002; accepted Dec. 11, 2002

U.S.B. was supported by a Senior Research Fellowship from the Wellcome Trust. S.S.D. received support from the National Centre for Biological Sciences/Tata Institute of Fundamental Research and a Kanwal Rekhi Fellowship. We thank Dr. J. J. Knierim for help with gold plating and electrode manufacture and Dr. A. D. Redish for the MClust software and advice on its use. We also thank Dr. M. M. Panicker for suggestions and comments on this manuscript.

Correspondence should be addressed to Upinder S. Bhalla, National Centre for Biological Sciences, Gandhi Krishi Vignana Kendra Campus, Bangalore 560065, India. E-mail: bhalla@ncbs.res.in.

Copyright $\odot 2003$ Society for Neuroscience $\quad 0270-6474 / 03 / 231903-13 \$ 15.00 / 0$
}

shows activation lasting $10-15 \mathrm{sec}$, followed by prolonged decrease in blood oxygen level-dependent (BOLD) signal in response to prolonged odor presentation. A similar activation time course is seen in piriform cortex. This indicates the possibility of functional interactions between these regions and their involvement in olfactory habituation. Electrophysiologically, the anterior piriform cortex has been shown to respond briefly to odor stimulation. It shows habituation after prolonged odor stimulation, leading to diminished response to subsequent stimulation (Wilson, 1998). Together, these findings implicate the HC and its inputs in the habituation process.

Hippocampal neurons have been shown to fire preferentially during port approach and cue sampling while learning tasks such as delayed-nonmatch-to-sample (DNMS) tasks are performed (Eichenbaum et al., 1991). This indicates hippocampal involvement in decision making. Recent DNMS studies have shown odor-specific responses of HC neurons (Wiebe and Staubli, 1999; Wood et al., 1999).

Data about basal response profiles would be valuable to gain an understanding of odorant representation in the $\mathrm{HC}$ in the absence of behavioral context. In this article, we look at responses of the hippocampal neurons to odors, in the absence of behavioral context, to establish their odor-response properties. Using anesthetized, freely breathing animals, we characterize the odor responses of neurons from the $\mathrm{OB}$ and $\mathrm{HC}$. We then look at their responses to repeated presentations of a given odor with varying time intervals between the presentations. We show that the odor responses of the hippocampal pyramidal cells are sensitive to the interodor air period (AP), suggesting involvement of the $\mathrm{HC}$ in temporal pattern discrimination and habituation. 


\section{Materials and Methods}

Animals and surgical procedures. All of the experimental procedures were approved by the National Centre for Biological Sciences institutional animal ethics committee, in accordance with the guidelines of the Government of India and equivalent guidelines of the Society for Neuroscience. Female Wistar rats 3-5 months old and weighing 190-225 gm (National Centre for Laboratory Animal Sciences, National Institute of Nutrition, Hyderabad, India) were anesthetized with a mixture of ketamine (100 mg/kg; Themis Chemicals, Mumbai, India) and xylazine (10 $\mathrm{mg} / \mathrm{kg}$; Indian Immunologicals, Hyderabad, India). Female rats were used because of their better survival during prolonged experiments. A surgical plane of anesthesia was maintained with thiopental sodium (3 $\mathrm{mg} / \mathrm{kg}$; Abbott Laboratories, Bombay, India) at $10 \mathrm{~min}$ after the first ketamine-xylazine injection and later as required. The surgical plane of anesthesia was judged by lack of foot withdrawal reflex or increased respiration rate in response to toe pinch and eye blink in response to touching the upper eyelid with a brush. The rectal temperature of the rat was maintained at $\sim 37^{\circ} \mathrm{C}$ with a heating pad during the surgery and an infrared lamp during recordings to reduce electrical noise. Respiration was monitored with a fine $(<200 \mu \mathrm{m})$, insulated thermocouple inserted in the ipsilateral nostril. The thermocouple signal was amplified 1000fold and stored on the computer after digitization at $300 \mathrm{~Hz}$. Exposures of $\sim 1.5 \mathrm{~mm}$ diameter were drilled over the $\mathrm{OB}(+8 \mathrm{~mm}$ anteroposterior, 1 $\mathrm{mm}$ lateral from bregma) and $\mathrm{HC}(-3.5 \mathrm{~mm}$ anteroposterior, $2 \mathrm{~mm}$ lateral for CA1 or $-3.5 \mathrm{~mm}$ anteroposterior, $3.5 \mathrm{~mm}$ lateral for CA3 recordings.). The dura was cut with iridectomy scissors and reflected.

Simultaneous extracellular recordings from the $O B$ and the $H C$ of anesthetized rats. Tetrodes (McNaughton et al., 1983; Recce and O'Keefe, 1989) were constructed using $\sim 18$ - $\mu$ m-diameter nichrome wire with $\sim 3.5$ - $\mu \mathrm{m}$-thick Formvar insulation (Diamond General Development Corporation, Ann Arbor, MI). The electrode tips were gold plated with noncyanide gold plating solution (SIFCO Selective Plating, Cleveland, $\mathrm{OH}$ ) to final tip impedance of $150-250 \mathrm{k} \Omega$ at $1000 \mathrm{~Hz}$. The tetrodes were inserted in the $\mathrm{OB}$ and the $\mathrm{HC}$ with micromanipulators mounted parallel to each other on a stereotaxic apparatus (INCO, Ambala, India). The exposures were covered with silicone grease after the electrodes had been pushed under the surface of the brain to stabilize the recordings and to prevent drying. The $\mathrm{OB}(+8 \mathrm{~mm}$ anteroposterior, $1 \mathrm{~mm}$ lateral) tetrode was advanced until an isolatable single unit was picked up in the mitral cell layer, characterized by a high background activity synchronous with respiration. Most of the recordings were from the lateral or ventral regions of the mitral cell layer. For CA1 recordings, tetrodes were lowered into the cell body layer of CA1 ( $-3.5 \mathrm{~mm}$ anteroposterior, $2 \mathrm{~mm}$ lateral, $\sim 2 \mathrm{~mm}$ deep) by monitoring for the typical bursting activity of pyramidal neurons with an oscilloscope and an audio monitor. Field potential responses to fimbria fornix stimulation were used to confirm the position of recording electrodes in the CA1 cell body layer in the initial recordings. For CA3 recordings, the electrodes were lowered into the cell body layer of CA3 $(-3.5 \mathrm{~mm}$ anteroposterior, $3.5 \mathrm{~mm}$ lateral, $\sim 3.3 \mathrm{~mm}$ deep) after passing through the CA1/2 ( 2.4 mm deep) cell body layer. CA3 pyramidal neurons show a typical nonaccommodating burst pattern, with a small reduction in recorded spike amplitudes, as against accommodating bursts of CA1/2 pyramidal cells, with considerable reduction in spike amplitudes during a burst.

The signals were amplified 10,000-fold and band-pass filtered between $300 \mathrm{~Hz}$ and $6 \mathrm{kHz}$. The amplified signals were digitized at 20 or $30 \mathrm{kHz}$ by use of a DAP3200e data acquisition card (Microstar Laboratories, Bellevue, WA) controlled with custom-written software. Every time the signal on one of the electrodes crossed a preset threshold, 32 prethreshold and 32 postthreshold samples (at 20 or $30 \mathrm{kHz}$ ) were stored from all of the simultaneously recorded channels on a tetrode.

We were using a six-channel amplifier for these recordings. Hence, we could not record from all of the channels on the two tetrodes. We recorded from three channels from the tetrode in the OB and three channels from the tetrode in the $\mathrm{HC}$. The positions of the electrodes were fine tuned such that at least two of the three HC electrodes picked up spikes with $>200 \mu \mathrm{V}$ peak to peak, and there was at least one isolatable unit on the OB electrodes. Generally, the background in the mitral cell body layer was very high, and therefore only the spikes having signal amplitude of $>300 \mu \mathrm{V}$ peak to peak could be isolated. Use of three electrodes instead of four may reduce the number of isolatable units, especially in the HC, but it did not hamper the unit classification, as judged by the refractory period of $>2 \mathrm{msec}$ and tightness of clusters.

At the end of the experiment, the recording sites were lesioned electrolytically. The brains were cryosectioned and stained with cresyl violet to locate lesions at the recording sites. All of the CA1 and CA3 and most of the $\mathrm{OB}$ recording sites were confirmed by lesioning. Some of the $\mathrm{OB}$ recording sites, especially the ones from the dorsal mitral cell body layer, could not be confirmed with lesioning because of damage to the dorsal surface of the bulb during removal from the skull. However, the background activity in the mitral cell body layer, as confirmed by the other lesions, was very distinctive. This supports the identification of the recorded cells as mitral-tufted cells. Figure $1 A$ shows the location of recording sites in the $\mathrm{OB}$ and the HC. Figure $1 B-D$ shows extracellular recordings from the $\mathrm{OB}$ and the $\mathrm{HC}$.

Odor delivery. The following odors were used for the experiments: isoamyl acetate (Ranbaxy Fine Chemicals, New Delhi, India), 1,4cineole, (+)-limonene (both from Sigma, St. Louis, MO), methyl salicylate (Polykem, Salem, India), and xylene (Qualigens, Mumbai, India).

Microcentrifuge tubes filled with odors were opened in front of the rat's nose (within $5 \mathrm{~cm}$ ) for study of the odor responsivity and selectivity of neurons from the OB and HC. The experimental rats had never encountered these odors before the experiment.

For delivering fixed concentrations of odors and for studying the effect of habituation, odorized air from an air dilution olfactometer was blown at the rat's nose. Odorized air blew at $30 \mathrm{l} / \mathrm{min}$ from a 0.25 -inch-diameter tubing placed $\sim 10 \mathrm{~cm}$ in front of the rat's nose. The design of the olfactometer was similar to the one described by Bhalla and Bower (1997), except that nitrogen was bubbled through the odors to prevent oxidation of the odors. Briefly, compressed air was filtered to remove oil and moisture and then passed through an activated charcoal filter to remove organic or odorous molecules. Purified air was bubbled through doubledistilled water to saturate it with water vapor. The air thus processed was used for reference and dilution purposes. Nitrogen (99.95\% pure; cylinders from Praxair, Bangalore, India) was bubbled through pure odor, and the saturated odor vapor so generated was diluted with air. Throughout the experiment, the nitrogen flow rate was maintained at $11 / \mathrm{min}$, and air flow rate was at $29 \mathrm{l} / \mathrm{min}$ using inline flow meters and needle valves. This generated a 1:30 dilution of saturated odor vapor, which was blown at the rat's nose from a tube located within $10 \mathrm{~cm}$ of the nostrils.

Air flow rate was briefly increased to $35 \mathrm{l} / \mathrm{min}$ during purge to quickly clear the odor from the dilution manifold and delivery tubing. Solenoid valves with fast $(<50 \mathrm{msec})$ switching time controlled the air and odor delivery. The solenoid valves were computer controlled. Odor solenoid turn-on time was monitored at $3.3 \mathrm{msec}$ resolution by digitizing (at 300 $\mathrm{Hz}$ ) and storing the computer output that controls the odor solenoid. The dead volume in the odor delivery tube and dilution manifold running from the olfactometer to the rat was $<70 \mathrm{ml}$. Hence, the estimated dead time of odor delivery at $30 \mathrm{l} / \mathrm{min}$ flow rate was $\sim 140 \mathrm{msec}$. This is corroborated by the response time of some of the odor-responsive neurons in the OB, which is of the order of $200 \mathrm{msec}$ after onset of odor delivery.

Each odor run consisted of cycles of air-odor-purge repeated multiple times: 4-12 trials for microcentrifuge tube delivery and 7-30 trials for olfactometer delivery. Odor period was fixed at $10 \mathrm{sec}$ and purge at $2 \mathrm{sec}$. $\mathrm{AP}$ was fixed for the given run. For odor delivery from microcentrifuge tubes, the interval between consecutive odor trials was $40 \mathrm{sec}$, whereas for olfactometer odor delivery, the consecutive runs had APs of 148, 98, 48, 18 , and $8 \mathrm{sec}$, with at least $15 \mathrm{~min}$ break between consecutive odor runs. Thus, the intervals between the start of successive odor presentation periods in a run were 160, 110, 60, 30, and $20 \mathrm{sec}$. For analysis of the effect of AP within a run on responses of the recorded neurons, the first odor trial was eliminated, so that preceding odor presentation occurred before the same interval for all of the odor trials in the run. Figure $2 \mathrm{~A}$ shows the schematic of the recording and odor delivery setup. Figure $2 B$ shows the odor delivery paradigm used for all experiments.

Data analysis. Putative single-neuron activities were classified by use of 
A
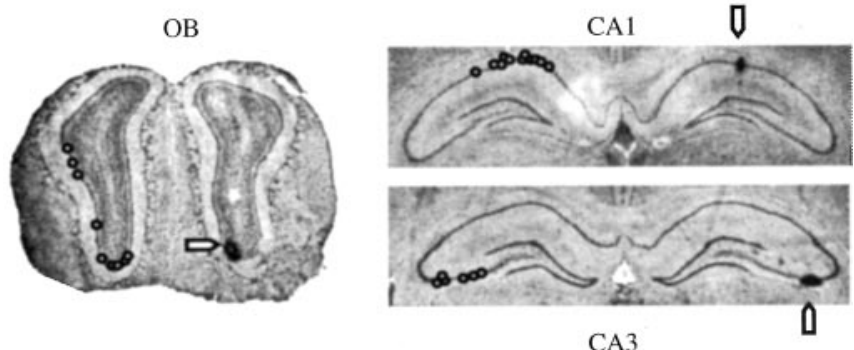

$\mathrm{CA} 3$

B

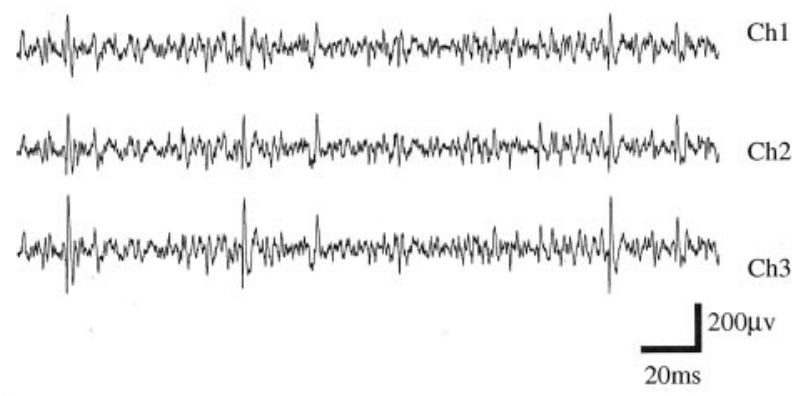

C
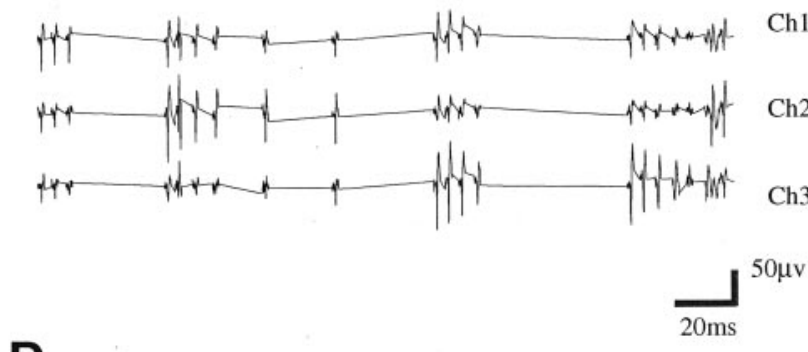

D

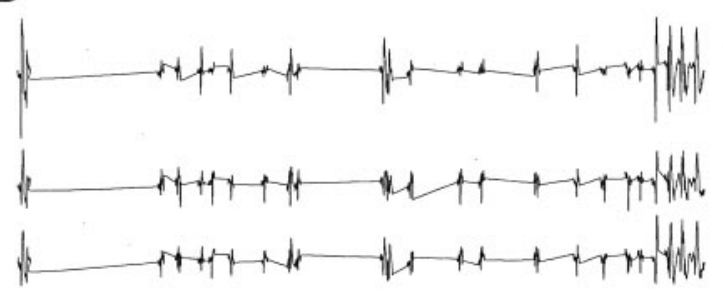

لـ

$100 \mu \mathrm{v}$

Figure 1. Extracellular recordings from $\mathrm{OB}$ and $\mathrm{HC} . A$, Lesions marking recording sites in $\mathrm{OB}$ mitral cell body layer $(O B)$ and hippocampal $C A 1$ and $C A 3$ pyramidal cell layer. Arrows indicate recording site lesions. Open circles on the contralateral side indicate the location of lesions from other recordings. $B$, Continuous extracellular recording from $0 \mathrm{~B}$. The high background activity is characteristic of mitral-tufted cell body layer. $C h 1, C h 2$, and $C h 3$ are simultaneously recorded channels from a tetrode. C, Triggered extracellular recordings from CA1 pyramidal cell layer. D, Triggered extracellular recordings from CA3 pyramidal cell body layer. Triggered recordings from $O B, C A 1$, and $C A 3$ were used for cluster cutting and additional analysis. For details, see Materials and Methods.

manual cluster cutting programs: xcut (custom software developed inhouse) and MClust (A. D. Redish; http://www.cbc.umn.edu/ redish/ mclust/). Waveform discrimination was based on evaluation of various parameters for every spike recorded simultaneously on the tetrode. Well defined cluster boundaries and the presence of a distinct refractory period as seen in the interspike interval histogram for the neuron were used as criteria for well isolated clusters. Timing of the individual action potentials corresponding to single units was used for additional analysis.

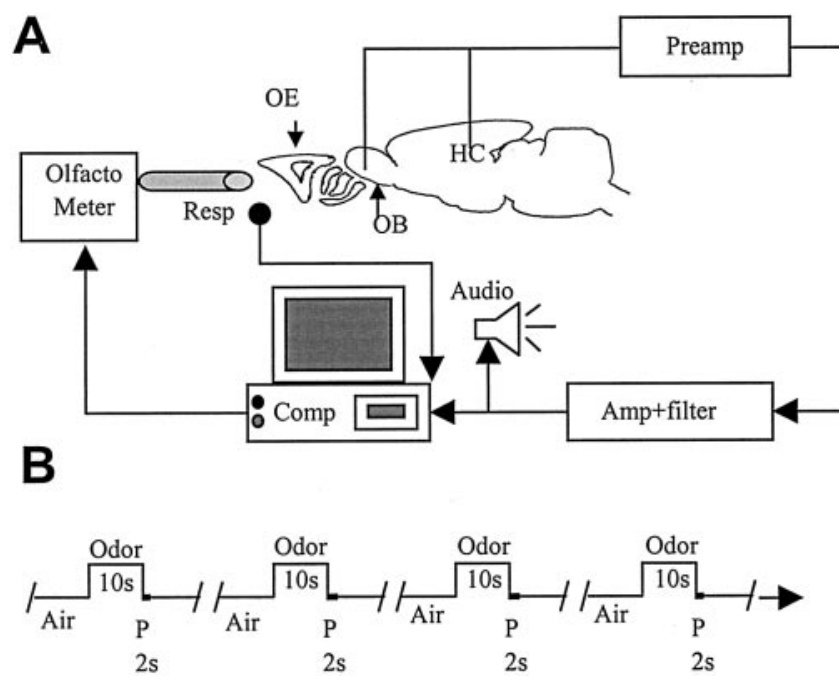

Figure 2. Experimental setup. $A$, Schematic representation of the experimental setup. Olfactometer, Olfactometer used for odor delivery; Resp, respiration monitor; Amp, amplifier; Audio, audio monitor for listening to extracellular recordings; $O B$, approximate location of $O B$ recording electrode; $H C$, approximate location of $H C$ recording electrode; $O E$, olfactory epithelium. $B, 0$ dor delivery paradigm. Odor duration was fixed at $10 \mathrm{sec}$, whereas purge $(P)$ was performed for $2 \mathrm{sec}$. AP was fixed for the given run, which consisted of multiple (up to 30) odor runs. There was a gap of $\geq 15$ min between consecutive runs of decreasing APs.

Only the neurons with basal firing rates $>0.1 \mathrm{~Hz}$ were included in the analysis.

Data were visualized by use of dot rasters and histograms. We then used statistical analyses on all of the neurons meeting the minimal firing rate criterion for characterizing response patterns of the $\mathrm{OB}$ and the $\mathrm{HC}$ neurons. One-way ANOVA was used to characterize the firing rate changes, and one-way multivariate ANOVA (MANOVA) was used to characterize the respiration tuning changes in response to odor presentation. The $p$ values given by these tests were used for comparing odor responses of the neurons. We elaborate the data analysis methods below.

Peristimulus-onset dot rasters and peristimulus time histograms were generated for preliminary odor-response analysis. Anesthetized rats used in these experiments had fairly regular respiration rates, of the order of one cycle per second. However, the odor onset time was not synchronized with respiration. Thus, there could be a variable delay of up to $1 \mathrm{sec}$ from odor delivery onset to the first inspiration after the odor onset, which would be the actual stimulus onset time. Odor onset time was thus corrected for the first inspiration after the odor delivery was turned on to align the stimulus onset time with more precision. This was used to generate respiration onset-corrected peristimulus-onset dot rasters (ROC-Raster) and respiration onset-corrected peristimulus time histograms (ROCPSTH). Figure 3 shows how respiration onset correction enables us to align the start of the stimulus, as well as making the temporal response pattern apparent in the ROC-Raster.

The spikes occurring during a $30 \mathrm{sec}$ period ( $10 \mathrm{sec}$ preodor plus $10 \mathrm{sec}$ odor plus $10 \mathrm{sec}$ postodor) were binned in 1 or $5 \mathrm{sec}$ bins for each odor trial within the run. This generated a matrix, with rows corresponding to consecutive odor trials within the run and columns corresponding to peristimulus time bin. For example, for the $5 \mathrm{sec}$ bin width, there were six columns corresponding to -10 to $-5 \mathrm{sec},-5$ to $0 \mathrm{sec}, 0$ to $5 \mathrm{sec}, 5$ to 10 $\mathrm{sec}, 10$ to $15 \mathrm{sec}$, and 15 to $20 \mathrm{sec}, 0$ being the respiration onset-corrected odor onset time. The number of rows corresponded to the number of trials. One-way ANOVA (MATLAB; MathWorks, Natick, MA) was then used to check the null hypothesis that the means of all of the columns are identical. A value of $p<0.05$ was used as a criterion for rejecting the null hypothesis. If, for the given run, the firing pattern of the neuron did not satisfy the null hypothesis, it was classified as a response to odor. Lack of significant variation in the preceding AP was confirmed for the runs with longer interodor intervals $(148,98$, and $48 \mathrm{sec})$. At shorter intervals of 18 and $8 \mathrm{sec}$, the air duration was expected to involve both postodor re- 

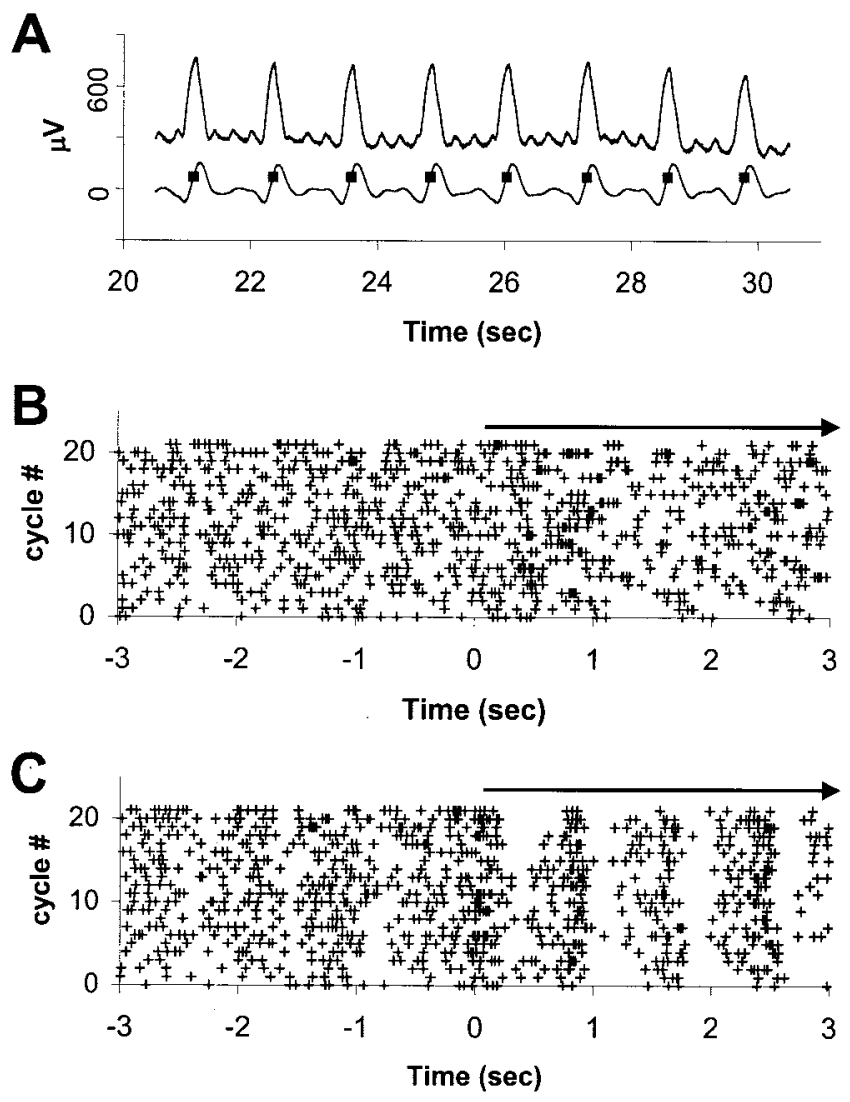

Figure 3. Effect of respiration onset correction on observed temporal dynamics of odor response of an $0 B$ mitral-tufted cell. $A$, Respiration trace recorded with thermocouple. Top trace shows the raw data. Bottom trace is smoothed trace with baseline nulling. Squares mark the points of maximum slope, corresponding to inhalation. Start of respiratory cycle was considered to be $200 \mathrm{msec}$ before this point. For details, see Material and Methods. B, Peristimulus onset dot raster, with 0 corresponding to the onset of odor delivery. Each horizontal line of + symbols corresponds to spikes occurring within the given trial. C, ROC-Raster for the same trial and the same neuron, with 0 corresponding to the first inhalation after odor onset. Arrows at top mark the odor period. Notice the temporal dynamics of responses, which become apparent on respiration onset correction. In this case, the neuron shows strong respiration phase tuning during odor presentation.

bound and basal activity. Hence, variability was not analyzed for these short intervals.

Respiratory phase tuning of the firing patterns and the changes in respiration tuning during odor presentation were analyzed. For this, the respiration trace recorded from the thermocouple was smoothed using a moving average, and the region of maximum slope was used to identify the start of the respiratory cycle (Fig. 3A). Inhalation corresponded to increase in voltage signal from the thermocouple. The onset of inhalation of each respiratory cycle was assigned a value of 0 , and the end of the cycle was assigned a value of 1 . Intermediate phases were assigned values linearly between 0 and 1 . For generating peristimulus respiratory phase raster (PSRPR), each spike was assigned a $y$ coordinate corresponding to the phase of respiration at the time of occurrence of the spike. Time of occurrence of the spike with respect to respiration onset-corrected odor onset time served as the $x$ coordinate (Bhalla and Bower, 1997; Wilson, 1998). Dot rasters so generated for consecutive odor trials within the run were overlaid to enable us to look at the respiratory phase preference for firing of the given neuron. For generating peristimulus respiratory phase histograms (PSRPH), each odor trial was divided into 5 sec epochs $(-10$ to $-5 \mathrm{sec},-5$ to $0 \mathrm{sec}, 0$ to $5 \mathrm{sec}, 5$ to $10 \mathrm{sec}, 10$ to $15 \mathrm{sec}$, and 15 to $20 \mathrm{sec}$, odor presentation being from 0 to $10 \mathrm{sec}$ ). Within each epoch, each respiratory cycle was subdivided into six equal bins, and each spike was summed into the bin corresponding to the phase of the respiratory cycle during which it occurred. The shape of this six-bin histogram corre-
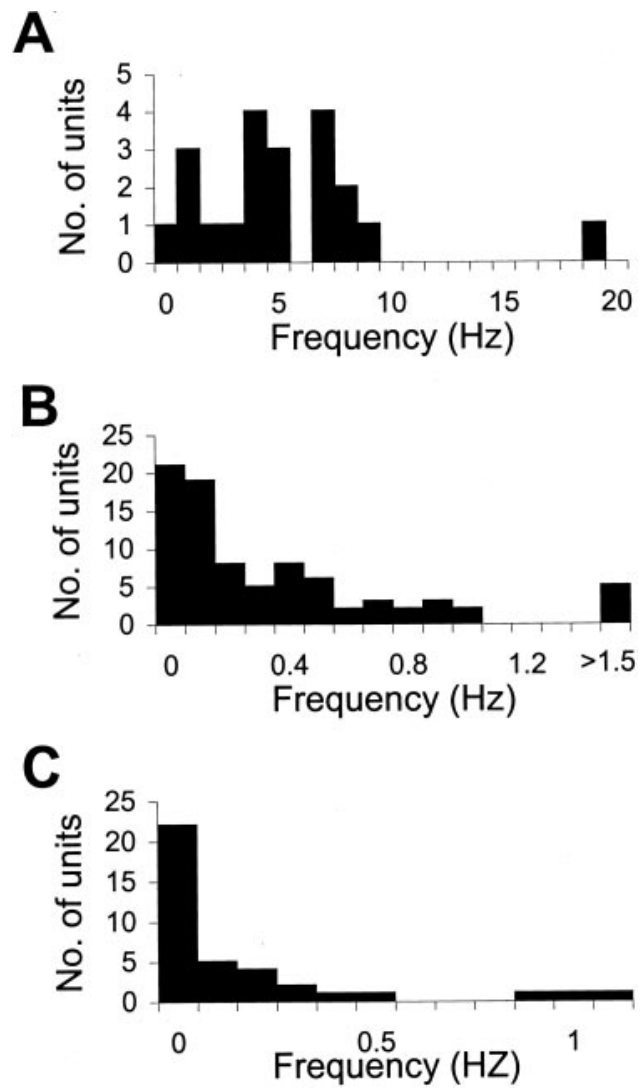

Figure 4. Basal firing rates of $O B$ mitral-tufted cells $(A)$, $C A 1$ pyramidal cells $(B)$, and $C A 3$ pyramidal cells ( $C$. The firing rates during 5 sec preceding odor delivery for all trials for all APs were averaged to estimate basal firing rate.

sponding to odor and purge epochs was compared with the immediately preceding AP. A change in the shape of the histogram is indicative of respiration tuning change. One-way MANOVA (MATLAB) was used to compare the histogram shapes. A value of $p<0.05$ was taken to be significant.

All $\mathrm{OB}$ and $\mathrm{HC}$ neurons were subjected to one-way ANOVA for determining firing rate responses and to one-way MANOVA for determining respiratory tuning changes in response to odor presentations at all APs. In case both ANOVA and MANOVA gave significant results, the MANOVA test was repeated after normalizing the firing rates of the air, odor, and purge periods. This was done because a large overall increase or decrease in the firing rate affects the MANOVA results. However, this was required primarily in the case of $\mathrm{OB}$ units, which tend to exhibit strong odor tuning changes, which are statistically significant even after normalization. A few CA1 $(n=2)$ and CA3 $(n=3)$ neurons also gave positive results in both the ANOVA and MANOVA tests and needed to be subjected to MANOVA after normalization.

\section{Results}

We recorded single neuron activities from either CA1 or CA3 pyramidal cell body layers simultaneously with neurons from the OB mitral cell layer from freely breathing anesthetized animals and examined their responses to odor stimulation.

\section{Basic responses}

Basal firing rates of $\mathrm{OB}$ mitral-tufted cells ranged from 0.6 to 20 $\mathrm{Hz}$ (mean $\pm \mathrm{SD}, 5.7 \pm 4.1 \mathrm{~Hz}$ ). The basal firing rates of HC CA1 pyramidal cells ranged from 0 to $3.7 \mathrm{~Hz}$ (mean $\pm \mathrm{SD}, 0.57 \pm 0.62$ $\mathrm{Hz}$ ), and those of CA3 pyramidal cells ranged from 0 to $1.1 \mathrm{~Hz}$ (mean \pm SD, $0.45 \pm 0.33 \mathrm{~Hz}$ ) (Fig. 4). Only the neurons with firing rates $>0.1 \mathrm{~Hz}$ were included in the analysis. Experiments 
described here were performed with an olfactometer to delivered a fixed concentration of odor with precise timing control.

To establish OB mitral-tufted cell and HC pyramidal cell responses to odors, we compared firing rates during the odor period and the postodor period with the immediately preceding AP using one-way ANOVA. OB mitral and tufted cells are known to show respiratory phase tuning, that is, to fire preferentially in certain phases of respiration and show a marked change in respiration phase tuning in response to odor presentation (Chaput et al., 1992). As described in Materials and Methods, we used MANOVA to compare respiration phase tuning between the air and odor periods and between the air and postodor periods. While using this as a measure for odor responses of mitral-tufted cells, we discovered that some of the HC pyramidal cells also showed significant respiratory tuning during odor presentation. Thus, along with firing rate changes, respiratory phase tuning changes were also used for characterizing odor responses from CA1 and CA3 along with OB. In all cases, a value of $p<0.05$ was categorized as a significant response.

Figure 5 shows firing rate and respiratory tuning changes recorded from OB mitral-tufted cells (Fig. 5A), CA1 pyramidal cells (Fig. $5 B$ ), and CA3 pyramidal cells (Fig. $5 C$ ). Figure $5 A-C, i$ and $i i$ (left half of figure) illustrates firing rate changes in response to odor stimulation using ROC-Raster and ROCPSTH, respectively. Figure $5 A-C$, iii and iv (right half of figure) illustrates respiratory coupling changes in response to odor stimulation using PSRPR and PSRPH. ROC-Raster, ROCPSTH, PSRPR, and PSRPH were generated as described in Materials and Methods. We consider the significance of respiration phase tuning responses in $\mathrm{HC}$ in Discussion.

The basal firing rate of the unit in Figure 5, $A i$ and $A i i$, is $\sim 20$ $\mathrm{Hz}$, which is on the higher end of spontaneous firing rates of mitral-tufted cells (Fig. 4). This neuron shows increased firing rate on odor onset, which remains high throughout the odor presentation. For $\sim 1 \mathrm{sec}$ after the end of odor presentation, it shows reduced firing and then rebounds. This is seen as an increased density of raster points from 0 to $10 \mathrm{sec}$ and reduced density just after $10 \mathrm{sec}$ in Figure 5Ai. In Figure 5Aii, this appears as a sustained increase in the height of $1 \mathrm{sec}$ bins during odor presentation.

Figure 5, Aiii and Aiv, shows a change in respiratory phase tuning of another mitral-tufted cell. This neuron shows a slight preference for the middle of the respiratory cycle before odor delivery. During odor delivery, the preference changes to the initial phase of respiration. The neuronal activity is turned off in the following phase $(0.2-0.6)$. This is seen as a thick cluster of points at the bottom and a near blank region in the middle of the PSRPR plot (Fig. 5Aiii) in the odor delivery period. In the PSRPH plot (Fig. 5Aiv), this appears as an increased spike count in the first respiration phase bin, followed by reduced count in the third and the fourth respiration phase bins during odor delivery. The respiratory tuning pattern reverts to the preodor pattern in a little more than $1 \mathrm{sec}$ after the end of odor delivery.

Firing rate changes of $\mathrm{OB}$ units in response to odor delivery showed a variety of profiles. These included a sustained increase in firing as shown in the example in Figure $5 A$, transient increases or decreases during the first 1-2 sec of odor delivery, poststimulus rebound, and other features. This range of responses of $\mathrm{OB}$ units is similar to that reported in many previous studies (Harrison and Scott, 1986; Hamilton and Kauer, 1989).

CA1 pyramidal cells have lower basal firing frequencies than $\mathrm{OB}$ mitral-tufted cells. Hence, the raster plots appear sparse compared with $\mathrm{OB}$ raster plots. Figure 5, Bi and Bii, shows a CA1 pyramidal cell responding to odor presentation by increasing its firing rate during the odor presentation. This is seen as higher density of raster points in the ROC-Raster (Fig. 5Bi) and increased height of $1 \mathrm{sec}$ firing rate bins in ROCPSTH (Fig. 5Bii). The increase in the firing rate is not sustained uniformly throughout the odor presentation. Some of the neurons $(n=3)$ in our data set respond by showing an increased firing rate on odor onset, coming back to baseline within 2-3 sec, whereas some others $(n=2)$ started responding only after 3-4 sec of odor delivery. Some neurons $(n=5)$ responded by a change in firing rate immediately after termination of odor delivery.

Respiration tuning change in the firing pattern of CA1 pyramidal cell is seen in Figure 5, Biii and Biv. Starting 2 sec into the odor delivery, the unit fires preferentially in the late phase of respiration and shows reduced firing at the end of the odor delivery. The change is clearly visible in the PSRPH also, although the start and the end of the respiration phase tuning change (from $\sim 2$ to $8 \mathrm{sec}$ ) does not exactly match the epochs ( 0 to 5 and 5 to 10 $\mathrm{sec}$ ) chosen to generate the plot.

Not all of the CA1 neurons showing respiration tuning change show a preference for the same phase of respiration. The duration of respiration tuning varied from neuron to neuron. Six neurons showed respiration tuning change in the $5 \mathrm{sec}$ epoch immediately after termination of odor delivery.

Most of the CA3 pyramidal cells tended to fire at a very low frequency, below $0.1 \mathrm{~Hz}$, and hence were excluded from the analysis. Eight of the 22 CA3 neurons meeting the minimum firing rate criteria showed odor responses.

Figure 5, Ci and $\mathrm{Cii}$, shows a neuron that shows a small but significant increase in the basal firing rate in the first second of odor presentation. Fig 5, Ciii and Civ, shows a respiration phase tuning change in response to odor presentation for another CA3 pyramidal cell. The unit is preferentially turned off during the middle part of the respiration cycle, during odor presentation.

Although we used firing rate changes and respiratory phase tuning changes as measures of odor responsiveness of the neurons, the temporal dynamics of their activation were observed to be quite complex. For example, some of the $\mathrm{OB}$ and $\mathrm{CA} 1$ neurons showed increased firing in the first $1-2 \mathrm{sec}$, followed by either return to baseline or inhibition below baseline level. For the purposes of this study, a response pattern was classified as a firing rate change in all cases in which the one-way ANOVA was significant. Although this is useful for differentiating between odorresponsive and nonresponsive neurons, the actual coding strategy of neurons may differ and is not examined in detail in this article. For example, most of the essential information could be coded for by the timing of the first spike after the stimulus or relative firing of an ensemble of neurons or even the timing of individual spikes (Petersen et al., 2001) rather than firing rate. It is also possible that our analysis has underestimated the number of hippocampal pyramidal cells responding to odor presentation with reduced firing rate. Low basal firing rates of these neurons coupled with stringent requirements of ANOVA would mean that "small" reductions in firing rates become characterized as nonsignificant. Thus, inhibition in HC neurons would be more amenable to study in awake animals with neurons showing higher firing rates.

\section{Responses of HC pyramidal cells and OB mitral-tufted cells to a range of odors}

We used a panel of five odors delivered from a microcentrifuge tube held near the anesthetized rat's nose to look for responses of $\mathrm{HC}$ and $\mathrm{OB}$ neurons to a range of odors. The aim of this set of 

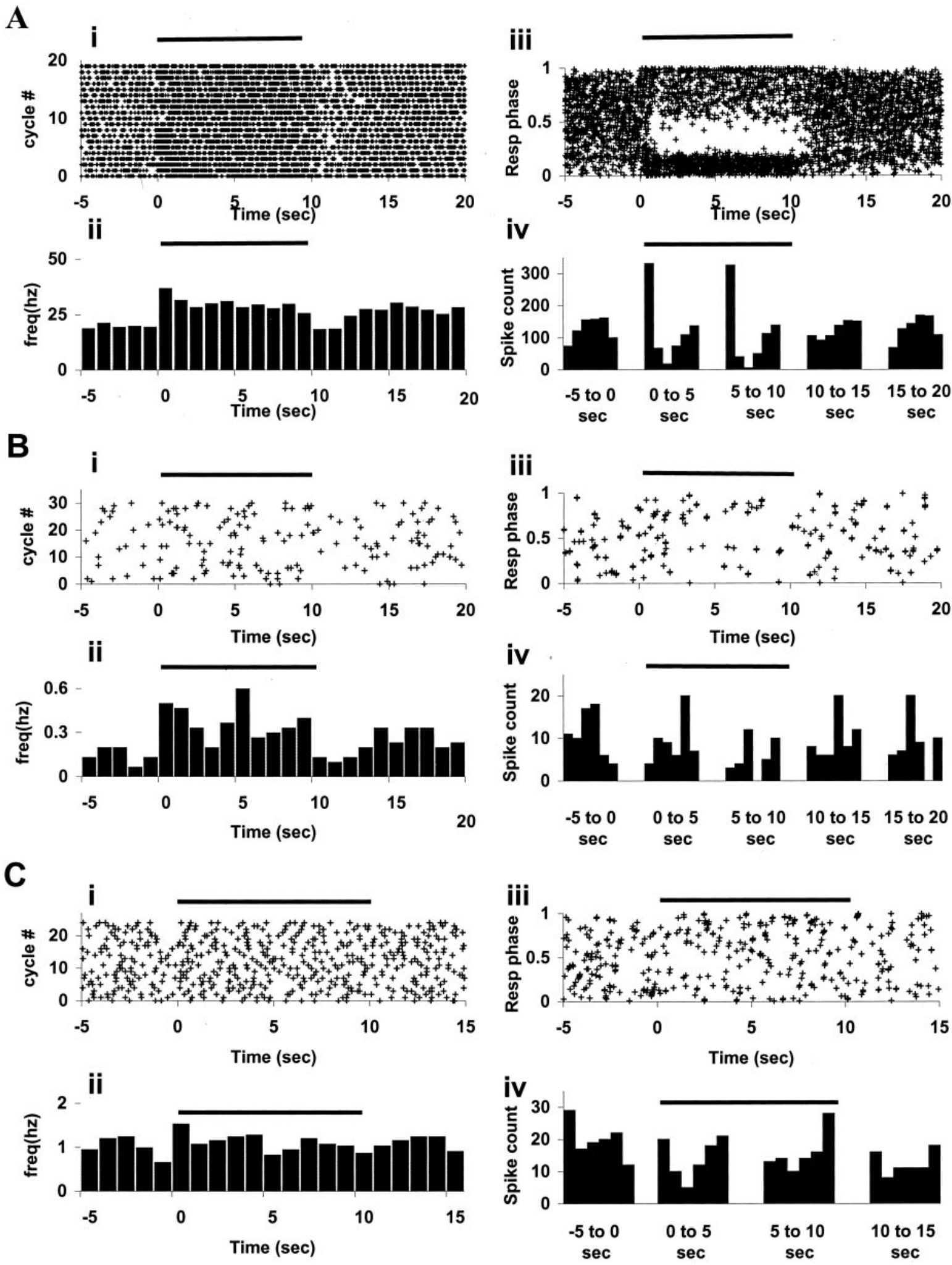

Figure 5. Odor response properties of neurons from $O B$ and $H C$. Horizontal line at top of each plot indicates the duration of odor delivery ( 10 sec, starting at 0 sec and going on until 10 sec). $A, O B$ mitral-tufted cells. Plots in $i$ and $i i$ are for neuron 16, and those in iii and $i v$ are for neuron 11. ROC-Raster ( $i$ ) and ROCPSTH (ii) show sustained firing rate increase in response to odor delivery ( $p<$ $10^{-15}$ ). Bin width for ROCPSTH is 1 sec. PSRPR (iii) and PSRPH (iv) show respiration tuning change in response to odor presentation $\left(p<10^{-15}\right)$. B, HC, CA1 pyramidal cells. Plots in $i$ and ii are for neuron 70, and those in iii and iv are for neuron 49. ROC-Raster ( $i$ ) and ROCPSTH (ii) (bin width 1 sec) show firing rate change in response to odor presentation ( $p=0.0036$ ). PSRPR (iii) and PSRPH (iv) show respiration tuning change during odor presentation ( $p=0.03$ ). C, HC, CA3 pyramidal cells. Plots in $i$ and $i i$ are for neuron 47, and those in iii and iv are for neuron 41. ROC-Raster ( $i$ ) and ROCPSTH (ii) (bin width $1 \mathrm{sec}$ ) show firing rate change during odor delivery ( $p=0.02$ ). PSRPR (iii) and PSRPH (iv) show respiratory tuning change during odor delivery ( $p=0.025$ ). Note that the time scale for C goes from -5 to $15 \mathrm{sec}$ only, because the odor runs were of the shortest duration: $8 \mathrm{sec}$ air, $10 \mathrm{sec}$ odor, and $2 \mathrm{sec}$ purge. For additional elaboration on the response properties of pyramidal cells from CA3, see Results. For details of analysis, see Materials and Methods. 


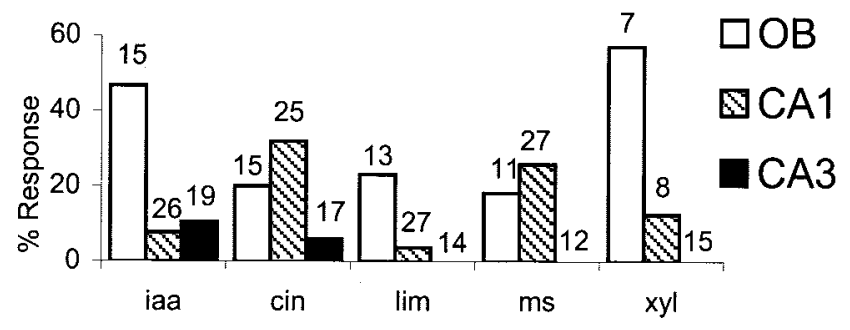

Figure 6. Percentage of $O B$ mitral-tufted cells and $H C C A 1$ and $C A 3$ pyramidal neurons responding to a panel of five odors. iaa, Isoamyl acetate; cin, 1,4-cineole; lim, (+)-limonene; $m s$, methyl salicylate; xyl, xylene. All odors were delivered manually. Numbers at top of each bin represent the total number of neurons tested. The ratio of responding neurons to total number of tested neurons was converted to percentage to facilitate comparison between sets with different number of neurons tested.

experiments was to quickly explore responses of $\mathrm{HC}$ and $\mathrm{OB}$ neurons to a range of odors before detailed temporal analysis. The odor panel consisted of isoamyl acetate, 1,4-cineole, (+)limonene, methyl salicylate, and xylene. The first four odors are pleasant odors, whereas xylene, an organic solvent, is an irritant and unpleasant odor, which could activate the trigeminal nerve along with providing olfactory stimulation. It was included in some of the recordings so as to enable comparisons with field potential studies showing CA3 responses to xylene and other organic solvents (Vanderwolf, 1992).

Each of the odors was presented between 4 and 12 times, with an interodor AP of $40 \mathrm{sec}$. Odor delivery time was $10 \mathrm{sec}$. Figure 6 shows percentage responses of the $\mathrm{OB}$ mitral-tufted and CA1 and CA3 pyramidal cells, as estimated using one-way ANOVA for firing rate changes and one-way MANOVA for respiratory tuning changes. The minimum firing rate criterion was applied for each odor, and therefore, the number of neurons included in the analysis varies slightly.

Isoamyl acetate and xylene gave rise to the largest number of responses from the mitral-tufted cells: 7 of 15 mitral-tufted cells tested in this manner responded to isoamyl acetate, whereas 4 of 7 cells responded to xylene. Xylene was included in only a subset of experiments because of its toxicity. CA1 pyramidal cells responded preferentially to 1,4-cineole ( 8 of 25 cells) and methyl salicylate ( 7 of 27 cells). In our sample set, only isoamyl acetate ( 2 of 19) and 1,4-cineole ( 1 of 17) elicited a response from CA3 pyramidal neurons. In all, 14 (of 28) CA1 and 3 (of 25) CA3 pyramidal cells responded to at least one of the odors. Of these, 4 CA1 neurons responded to two odors and one CA1 neuron responded to 3 odors. Other CA1 and CA3 neurons responded to only one odor. Thus, all of the odor-responsive neurons in this study show selectivity for odors. This selectivity for different odors exhibited by the CA1 pyramidal neurons points to a possible role for $\mathrm{HC}$ in odor recognition. It was not possible to control the onset precisely and turn off the odor delivered from tubes. The concentrations of the odors could also be expected to be variable. Thus, there is a possibility that the odor selectivity is influenced by variability in concentration and pattern of odor delivery in this stimulus protocol.

\section{Habituation: modulation of $\mathrm{HC}$ and $\mathrm{OB}$ odor responses by interval between odor stimuli}

To study the effect of a given odor trial on single-unit responses to subsequent trials of the same odor, we systematically went through a range of interodor APs of 148, 98, 48, 18, and $8 \mathrm{sec}$ using either isoamyl acetate or 1,4-cineole. In each case, the odor duration was $10 \mathrm{sec}$. The choice of odor was based on the results of the previous experiment using the panel of odors in many of the experiments. The time intervals were chosen to span previously used intervals and the receptor adaptation time course (Wilson, 1998). The main aim of this set of experiments was to characterize the response patterns of odor-responsive neurons and not to look at the percentage of the neurons responding to odor. This set of experiments was performed using an olfactometer to deliver fixed concentrations of odor with a precise timing. We started with a series of $10 \mathrm{sec}$ odor stimuli at the longest AP of $148 \mathrm{sec}$. After a break of at least $15 \mathrm{~min}$, we performed the multiple-odor trials at $98 \mathrm{sec}$. After a additional $15 \mathrm{~min}$ break, the odor was presented at an interval of $48 \mathrm{sec}$ and so on until an AP of $8 \mathrm{sec}$. We chose to follow the sequence of runs from longest to shortest APs such that expected habituation was less for earlier runs than later runs. Also, we expected the 15 min interval to be sufficient for habituation caused by previous odor run to wear off. The number of trials varied from 7 to 30. Approximately one-half of the runs were performed using $<10$ trials. Because the $p$ value estimation using one-way ANOVA and one-way MANOVA takes the number of trials (degrees of freedom) into account, they can be compared directly.

We pooled the data from the two odors, because there was no clear difference between the response patterns in any of the regions tested. Figure $7 A$ shows the percentage of neurons responding to odor presented at a range of APs, as estimated by one-way ANOVA for firing rate changes and one-way MANOVA for respiratory tuning changes. At least $50 \%$ of OB mitral-tufted cells responded to the odor presented at a given AP. This is on the higher side of the reported odor response percentage of OB mitral-tufted cells. Chaput et al. (1992) report a similar response percentage. As discussed by them, the percentage of neurons classified as responsive is a function of classification methods such as inclusion of respiratory tuning change or number of bins into which the respiratory cycle is divided. Another factor that might contribute to our observation of higher response percentage is the fact that most of our recordings were from a small region of the ventrolateral $\mathrm{OB}$. The response percentage in CA1 for different APs varied between 8 and 23\%, and that in CA3 was similar (Fig. 7A). However, most of the CA3 units recorded in this study fired at frequencies below $0.1 \mathrm{~Hz}$. These were not included in the analysis. Thus, the number of CA3 units included in this study is smaller than that of the CA1 units.

Seventeen of the $21 \mathrm{OB}$ mitral-tufted cells, 27 of 73 CA1 pyramidal cells, and 8 of 22 CA3 pyramidal cells showed a statistically significant odorant response at one or more APs, as measured using ANOVA. Interestingly, six of seven CA1 interneurons also responded to odor at one or more APs (Fig. $7 B$ ). A post hoc $Z$ test comparing odor period with immediately preceding AP was performed. The $Z$ scores so generated could also be used for direct comparisons of odor responses of $\mathrm{OB}$ and $\mathrm{HC}$ neurons with a large difference in their basal firing rates. $Z$ scores of neurons responding with firing rate changes (as determined by one-way ANOVA) varied from \pm 1.96 to \pm 11 in $\mathrm{OB}$, \pm 1.96 to \pm 3.8 in CA1, and \pm 1.96 to \pm 2.4 in CA3. A $Z$ score of 1.96 corresponds to a two-tailed probability of 0.05 , the significance cutoff used in this study. We find that the ANOVA tests described above are more stringent than the $Z$ scores, and we therefore base our comparisons on $p$ values derived from the ANOVA tests. Figure $7 D-G$ shows a comparative summary of significant responses in the various regions. A sizable fraction of OB mitral-tufted cells show highly significant responses $(p<$ 0.001 ), many of which are respiration tuned. None of the HC neurons show such significant responses. This difference could 

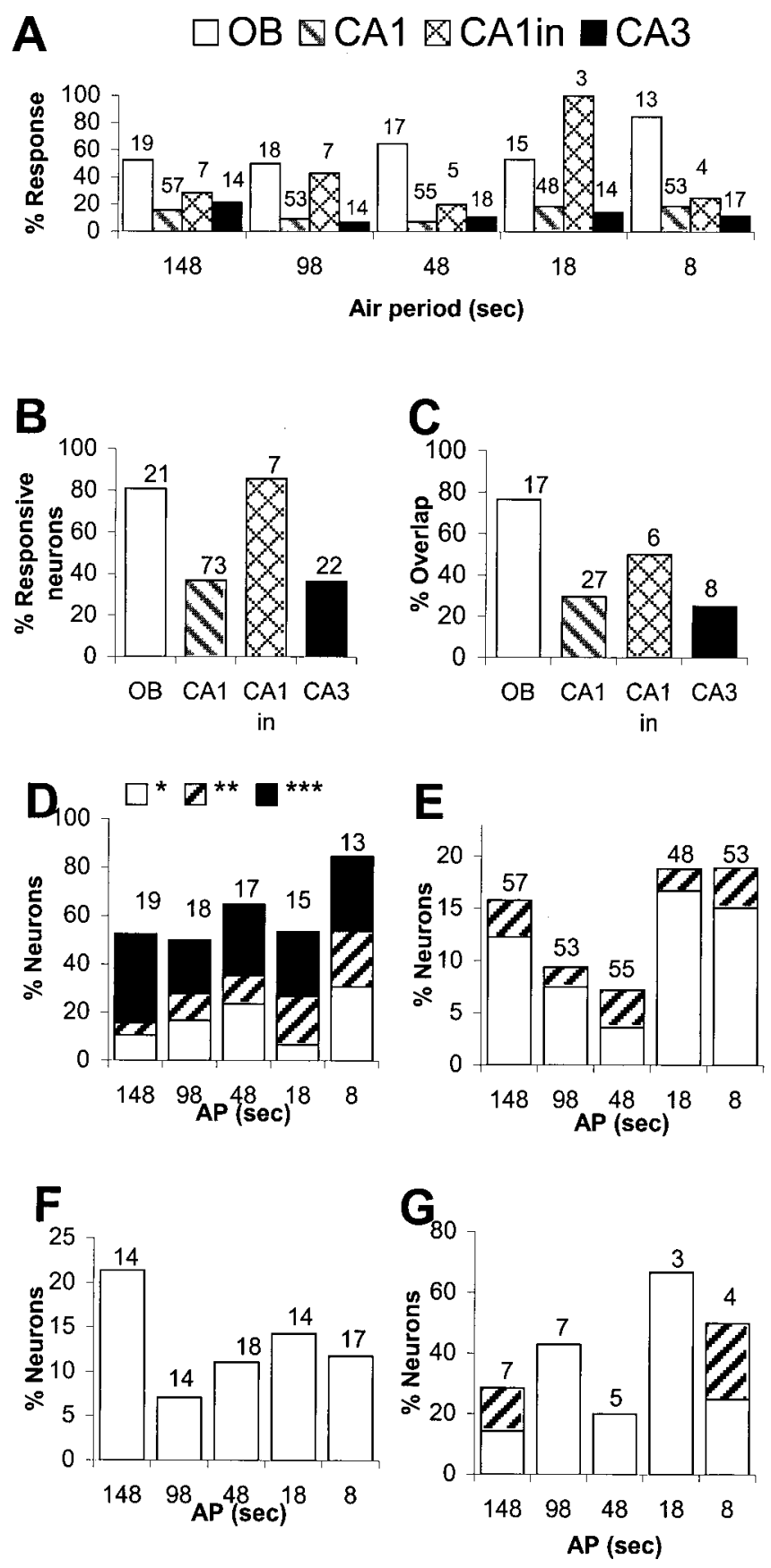

Figure 7. Odor response properties of $O B$ and $\mathrm{HC}$ neurons. $A$, Percentage of $O B$ mitral-tufted cells (OB), HC CA1 pyramidal cells (CA1), CA1 interneurons (CA1in), and CA3 pyramidal neurons (CA3) responding to odor delivered at different APs. Numbers at top of each bin represent total number of neurons tested. $B$, Percentage of neurons responding to odor during at least one of the five runs at different APs. Numbers at top of each bin are total numbers of neurons tested. $C$, Percentage of odor-responsive neurons that respond to odor delivered at more than one AP. Numbers at top of each bin are total number of neurons that respond to odor delivered at one or more APs. D-G compare the significance levels of responses, as estimated using ANOVA, to odors delivered at a range of APs. Blank portions of the bars indicate percentage of neurons responding at significance levels of $0.01<p<0.05\left(^{*}\right)$. Hatched portions of the bars indicate percentage of neurons responding at significance levels of $\left.0.001<p<0.011^{* *}\right)$. Solid portions of the bars correspond to percentage of neurons responding at significance levels of $p<0.001$ $(* * *)$. Total number of neurons, shown at top of each bin, is same as indicated in $A . D, O B$ mitral-tufted cells. E, CA1 pyramidal cells. F, CA3 pyramidal cells. G, CA1 interneurons. Note the different scales for neuronal response percentages in $D-G$. be caused partly by the large firing rate differences between the cell types. In addition, we suggest that the higher significance levels indicate that the $\mathrm{OB}$ neurons respond more strongly to odor presentation than HC neurons, which may be integrating data from other inputs.

Several (6 of 17) of the OB mitral-tufted cells responded to odor delivered at all APs tested (Fig. $8 \mathrm{~A}$ ), and a majority of mitral-tufted cells responded to odor at multiple APs (Figs. 7C, $8 A$ ). Eleven of 17 recorded neurons responded at some but not all APs. This dependence of responses on AP may be indicative of habituation or temporal selectivity, as elaborated in Discussion.

None of the CA1 units responded to odor at all APs, and a majority (19 of 27 odor-responsive neurons) responded only to odor delivered at a single AP (Figs. 7C, 8C). Three of the six odor-responsive CA1 interneurons responded to odor at more than one AP (Figs. 7C, 8D). In CA3 also, six of the eight odorresponsive neurons responded to odor at only one AP (Figs. $7 C$, $8 B$ ). There is no clear trend in odor response preferences of $\mathrm{OB}$ or HC units for different APs, as seen from Figures $7 A$ and 8.

Eight CA1 and two CA3 pyramidal cells gave statistically significant responses at more than one AP. Approximately one-half of these (five CA1 and one CA3) responded to nonadjacent APs. For example, CA1 unit 79 responded at 148,48 , and $8 \mathrm{sec}$ AP but not at 98 and $18 \mathrm{sec}$ (Fig. 8). CA1 neurons 82, 83, 84, and 86 were recorded simultaneously with neuron 79 (Fig. 8). Neurons 79 and 82 respond at nonadjacent APs, whereas the rest respond at only one AP. Thus, multiple response selectivities coexist. As considered in Discussion, these response properties suggest ensemble or higher-order recording schemes, which will require additional experimentation to resolve.

A large fraction of $\mathrm{OB}$ units showed simultaneous firing rate and respiratory tuning changes in response to odor delivery (Fig. $8 A$ ). Interestingly, a very small fraction of the $\mathrm{HC}$ neurons showed simultaneous firing rate and respiratory tuning changes in response to odor delivery (Fig. $8 B-D$ ).

We asked whether the level of anesthesia and status of the preparation at the time of the recording could influence odor responsiveness and hence apparent AP tuning. In four of the experiments, we repeated one of the APs at the end of the experiment. The units recorded in these experiments did not respond during either of the repeated runs of the AP. This does not provide a positive confirmation that neurons are consistently tuned to a specific AP but does not contradict the presence of such tuning. One of the CA3 pyramidal cells, not included in the study because of low basal firing rate $(0.05 \mathrm{~Hz})$, showed increased firing in response to odor at $98 \mathrm{sec} \mathrm{AP}$ in two runs spaced $3 \mathrm{hr}$ apart. We also performed several odor runs for a given AP for up to 30 trials. The odor responses in these cases were therefore monitored over a period of up to $80 \mathrm{~min}$, depending on AP. In several of these cases, we found statistically significant odor responses, again suggesting that AP tuning is not dependent on relatively short-term changes in state of the preparation. To address this issue conclusively, it may be necessary to repeat these experiments over multiple days in chronically implanted animals.

\section{Basis of AP selectivity}

What neural mechanisms underlie AP selectivity? We hypothesized that there could be three mechanisms operating to bring a neuron into or out of the selective state. First, the response amplitude or respiration tuning of the neuron could change as a function of AP. Second, the background activity could become larger or more variable depending on AP. Third, network effects may occur that make the odor responses more different from the 


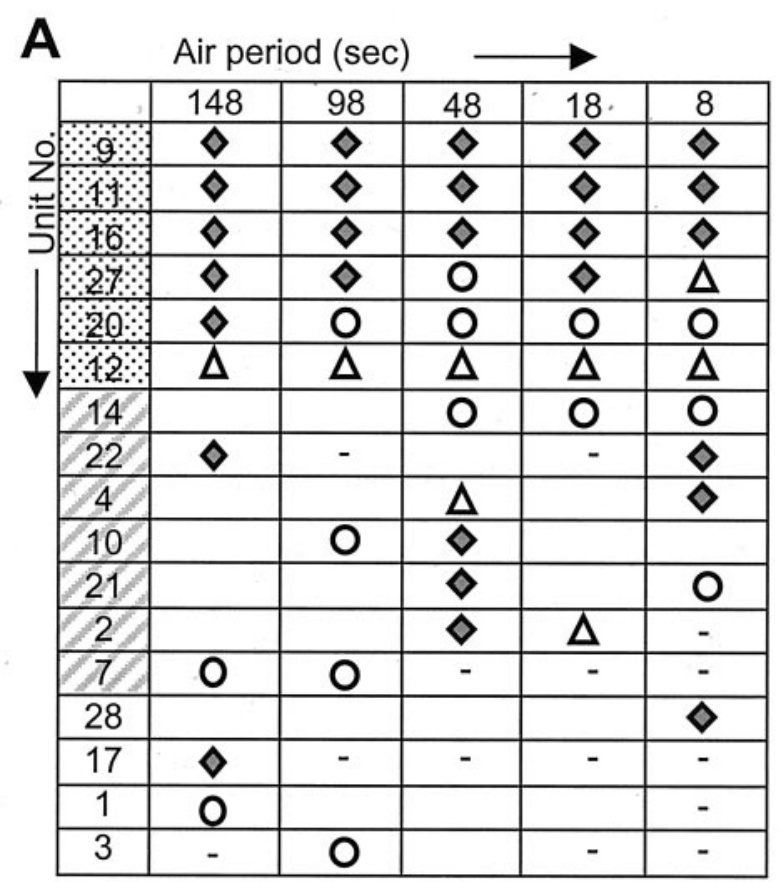

\begin{tabular}{|c|c|c|c|c|c|c|}
\hline \multirow[t]{2}{*}{ B } & \multicolumn{6}{|c|}{ Air period (sec) } \\
\hline & & 148 & 98 & 48 & 18 & 8 \\
\hline & 37 & $\diamond$ & $\diamond$ & & & \\
\hline & 21 & $\Delta$ & & 0 & & \\
\hline & 33 & $\Delta$ & & & & \\
\hline & 26 & & & $\Delta$ & & \\
\hline & 23 & & & & 0 & \\
\hline & 36 & & & & 0 & \\
\hline & 41 & - & - & & & $\Delta$ \\
\hline & 47 & - & - & - & - & $\Delta$ \\
\hline
\end{tabular}

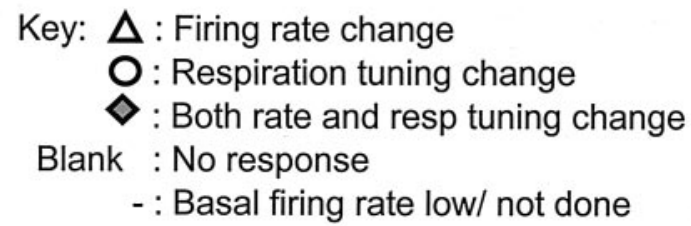

C Air period (sec)
\begin{tabular}{|c|c|c|c|c|c|}
\cline { 4 - 6 } & 148 & 98 & 48 & 18 & 8 \\
\hline 79 & $\Delta$ & & $\Delta$ & & O \\
\hline 70 & & O & O & $\Delta$ & \\
\hline 23 & 0 & & & $\checkmark$ & \\
\hline 82 & 0 & & & & O \\
\hline 8 & & $\Delta$ & & & $\Delta$ \\
\hline 72 & & & & $\Delta$ & $\Delta$ \\
\hline 12 & $\Delta$ & - & & & 0 \\
\hline 49 & $\Delta$ & 0 & & - & \\
\hline 53 & $\Delta$ & & & & \\
\hline 41 & $\Delta$ & & - & & \\
\hline 84 & 0 & & & & - \\
\hline 83 & $\Delta$ & & & - & \\
\hline 11 & & $\Delta$ & & & \\
\hline 52 & & $\Delta$ & & - & \\
\hline 30 & & & 0 & & \\
\hline 94 & & & 0 & - & - \\
\hline 21 & & & & 0 & \\
\hline 47 & - & & & $\Delta$ & \\
\hline 59 & & - & & $\Delta$ & \\
\hline 39 & & & - & $\Delta$ & \\
\hline 58 & - & - & & $\Delta$ & \\
\hline 44 & - & - & & $\Delta$ & \\
\hline 27 & & & & & $\Delta$ \\
\hline 28 & & & & & $\Delta$ \\
\hline 37 & & & & & $\Delta$ \\
\hline 13 & & & & & 0 \\
\hline 86 & & & & & 0 \\
\hline
\end{tabular}

\begin{tabular}{|c|c|c|c|c|c|c|}
\hline & & Air $p$ & $\mathrm{~d}$ (s & & $>$ & \\
\hline & & 148 & 98 & 48 & 18 & 8 \\
\hline & 36 & & 0 & & 0 & $\Delta$ \\
\hline & 69 & & $\Delta$ & & 0 & \\
\hline & 3 & 0 & & & & $\Delta$ \\
\hline & 89 & & & $\Delta$ & - & \\
\hline & 17 & & 0 & - & - & - \\
\hline & 18 & 0 & & - & - & - \\
\hline
\end{tabular}

Figure 8. Matrix of unit response against $A P$ for odor-responsive $O B, C A 1$, and $C A 3$ neurons. Only the neurons responding to the odor delivered at least one $A P$ are included. Units are rearranged so as to put the ones responding to odor at larger number of $A P s$ above the ones responding to lesser number of APs. A, OB mitral-tufted cells. B, CA3 pyramidal cells. C, CA1 pyramidal cells. D, CA1 interneurons. Units that responded to odor delivered at all APs are highlighted with dotted background. Units that responded to odor runs for more than one AP but not to all runs are highlighted with hatched background. Whereas $\mathrm{OB}$ shows predominance of units responding to odor over multiple APs, $\mathrm{HC}$ has predominantly units that respond preferentially to odor delivered at a single AP.

background activity but do not lead to a simple global effect, as suggested by the previous two possibilities. We tested the first two possibilities by analyzing the trends in trial-to-trial responses and variability.

The firing patterns of neurons included in this study vary from trial to trial. Figure 9 shows the raw data for one $\mathrm{OB}$ neuron and one CA1 pyramidal cell at two APs each. The OB neuron responds to the odor at both APs, but the pattern of response is different at the two APs. At $98 \mathrm{sec} \mathrm{AP}$, the response does not vary visibly from trial to trial, whereas at $18 \mathrm{sec} \mathrm{AP}$, the odor response changes from sustained increase in firing rate in initial odor trials to a transient increase in firing rates in the later trials (Fig. 9A). Thus, even the same neuron may exhibit significant responses that arise from different underlying temporal patterns of activity. The CA1 neuron responds to odor presentation by a decreased firing rate during odor followed by postodor rebound for odor presentation at $98 \mathrm{sec} \mathrm{AP}$, although it showed no response at 18 sec AP (Fig. 9B). Given the low firing rate of the neuron, it is 
A
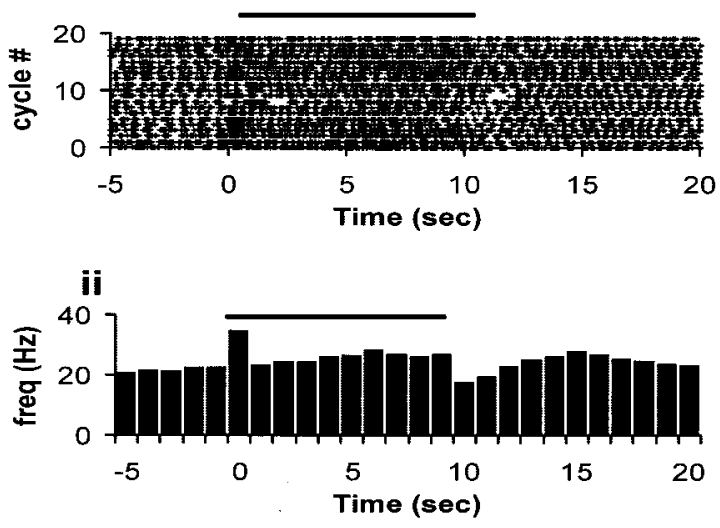

B
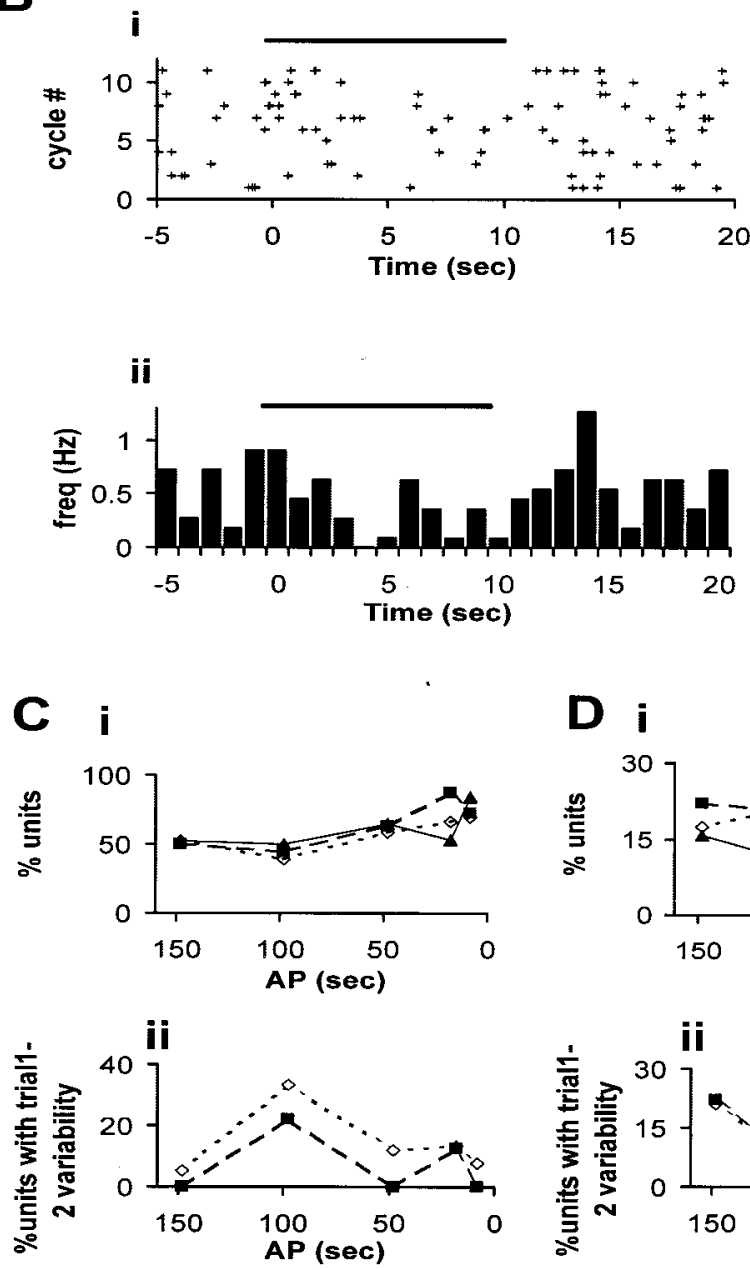

D i
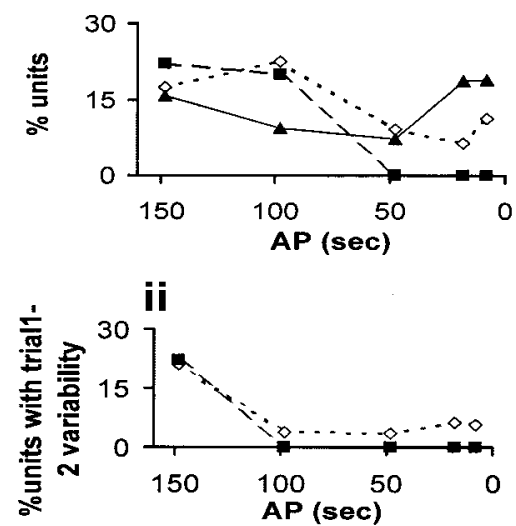
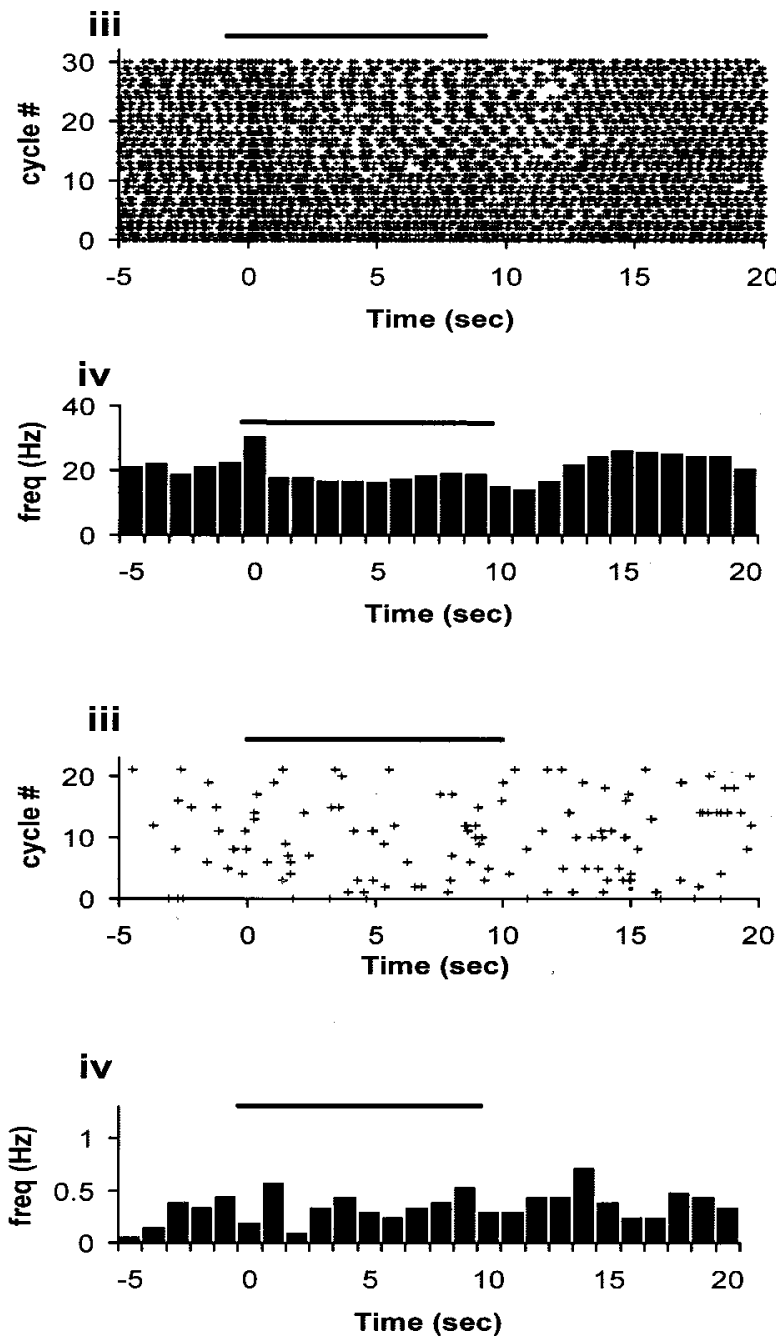

E i
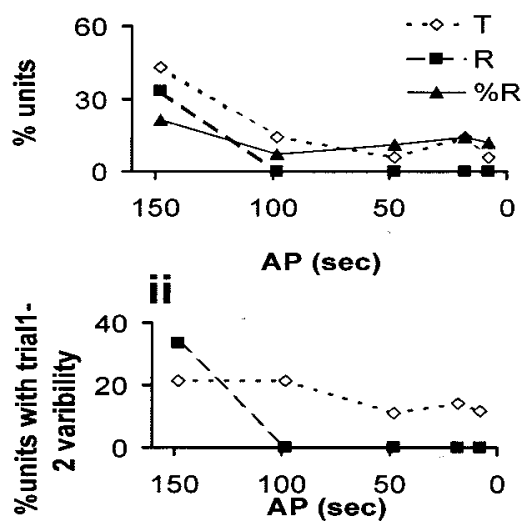

Figure 9. Variability in odor responses. A, ROC-Rasters and ROCPSTHs of OB mitral-tufted neuron 16 showing variability in its odor response as a function of AP. $i$, ii, AP, 98 sec; $i i, i v$, AP, 18 sec. The neuron gives statistically significant responses in both cases (one-way ANOVA; $p=1.9 \times 10^{-15}$ for 98 sec AP and $p=4 \times 10^{-14}$ for 18 sec AP) but shows higher variability at 18 sec AP, as seen from the ROCPSTH. The decrease in odor-stimulated firing in Aii is suggestive of habituation. B, ROC-Rasters and ROCPSTHs of a CA1 pyramidal cell responding to odor at 98 sec AP ( $i$ and $i i$ ) and not responding to the same odor at $18 \mathrm{sec}$ AP (iii and $i v)$. One-way ANOVA; $p=0.038$ and $p=0.7$, respectively. C $E$ show variability measured using two-way ANOVA. The percentage of odor-responsive neurons satisfying a criterion of $p<0.05$ is plotted for comparison. Open diamonds represent percentage of total ( $T$ ) neuronal population showing trial-to-trial variability. Filled squares correspond to percentage of odor-responsive $(R)$ neurons showing trial-to-trial variability. Filled triangles represent percentage of neurons showing statistically significant odor responses $(\% R)$. C, OB mitral-tufted cells. D, CA1 pyramidal cells. E, CA3 pyramidal cells. For C-E, $i$, Plot of percentage of neurons showing trial-to-trial variability as a function of AP for all trials starting from the second trial; $i i$, plot of percentage of neurons showing trial-to-trial variability between the first two trials as a function of AP. 
difficult to compare the response in consecutive trials by visual inspection of the dot rasters, and we rely on statistical methods for this comparison.

We performed post hoc two-way ANOVA to characterize trialto-trial variability within a run while simultaneously confirming the odor responsiveness of the neuron. As described in Materials and Methods, these comparisons excluded the first trial of each run, because it followed a long ( $15 \mathrm{~min}$ ) AP. Figure 9 also shows the trial-to-trial variability for $\mathrm{OB}$ mitral-tufted cells (Fig. 9C) and HC CA1 (Fig. 9D) and CA3 (Fig. 9E) pyramidal cells. The trial-to-trial variability measured using this two-way ANOVA is a composite of changes in basal firing patters and changes in odor response properties of the neurons over consecutive trials. We further compared the ratios of SD of firing rate with mean firing rates during the air, odor, and postodor periods (data not shown). There was no significant difference between the three periods, and this test seemed to be a less sensitive measure of variability than two-way ANOVA. We therefore suggest that the contribution to the variability measured by the ANOVA test is not coming disproportionately from any one of the three periods.

Figure $9 \mathrm{Ci}$ shows the percentage of $\mathrm{OB}$ mitral-tufted cells showing variability $(p<0.05)$. A larger number of odorresponsive units showed trial-to-trial variability at shorter APs (e.g., 8 of 11 at $8 \mathrm{sec}$ AP) than at the longer APs (e.g., 5 of 10 at 148 sec AP). A possible explanation for this is that habituation effects may be stronger at shorter APs than longer ones. The units that do not respond to odor presentation do not show any observable trend. Figure 9Cii shows the variability between the first and the second trials for each AP. There is no discernible trend in the percentage of neurons showing variability.

Figure 9, Di and Ei, shows the percentage of CA1 and CA3 pyramidal cells showing variability $(p<0.05)$ between all of the trials excluding the first trial. The overall percentage of neurons showing statistically significant trial-to-trial variability is lower in HC than OB. HC pyramidal neurons show higher trial-to-trial variability at longer APs than shorter APs. This trend is the reverse of the trend in the OB. This trend is seen in the case of both odor-responsive and nonresponsive neurons. Figure 9, Dii and Eii, shows the variability between the first and second trials for each AP for CA1 and CA3 pyramidal cells, respectively. The trend toward higher variability at longer APs is repeated for all CA1 cells and odor-responsive CA3 cells, but the CA3 cells that do not respond to odor presentation do not show any discernible trend. The trend in the percentage of HC neurons showing variability may suggest an effect of AP on overall activity in the HC. However, additional experiments are necessary to understand the physiological significance of this phenomenon.

We plot the percentage of neurons showing significant odor responses along with the percentage of neurons showing trial-totrial variability to compare odor response properties of the neurons and their trial-to-trial variability. We do not observe any correlation between the two (Fig. 9Ci, Di,Ei).

To summarize, our data suggest that the AP dependence of variability in neuronal responses is not sufficient to account for AP selectivity of hippocampal neurons. The AP dependence of variability in odor-selective and nonselective neurons is similar, and variability does not appear to be larger in air, odor, or postodor periods. This result argues against the first two possibilities, namely, a change in neuronal response amplitudes or changes in background activity, leading to change in the statistical significance of odor responses of the neurons. As discussed below, we suggest that more complex network effects lead to AP selectivity in HC neurons.

\section{Discussion}

Olfactory processing involves complex temporal dynamics, including stimulus patterning, respiratory sampling, and habituation. Here, we examine representation of odor identity and time in $\mathrm{HC}$ with reference to simultaneously recorded responses from $\mathrm{OB}$. HC is a higher-order integrative area of the brain, known to be involved in representation of space and context, although the exact nature of representation is controversial (Eichenbaum, 2000; Redish, 2001). We performed simultaneous OB and HC single-unit recordings from anesthetized, freely breathing rats. Responses of $\mathrm{HC}$ to a panel of five odors were studied to analyze odor specificity of $\mathrm{HC}$ response. We then studied the effect of the time interval between repeated odor stimuli on $\mathrm{HC}$ and $\mathrm{OB}$ responses by repeatedly presenting odor at a range of APs.

\section{Odor response profile of hippocampal neurons}

Electrical stimulation of OB (Yokota et al., 1967) or piriform cortex (Habets et al., 1980) leads to activation of HC pyramidal cells. A small fraction of CA1 pyramidal cells show odor-specific activation while performing DNMS tasks (Wiebe and Staubli, 1999; Wood et al., 1999). Our experiments examine the neural substrate for these observations by demonstrating responses of $\mathrm{HC}$ neurons to odor stimulation in the absence of behavioral context. We observe a much higher fraction (48\% in CA1, 12\% in CA3) of odor-responsive neurons in $\mathrm{HC}$ than have been reported previously.

Single-unit recordings from rats show that up to $70 \%$ of recorded HC neurons are activated while performing DNMS tasks (Wiebe and Staubli, 1999; Wood et al., 1999). In these studies, $\sim 10 \%$ of all recorded cells from CA1 show odor selectivity while performing DNMS tasks. We examined the hypothesis that odor selectivity is exhibited inherently by $\mathrm{HC}$ neurons and provides olfactory context information for complex tasks such as DNMS. Forty-eight percent of CA1 pyramidal cells and $12 \%$ of CA3 neurons responded selectively to a subset of presented odors. Thus, our data indicate that odor selectivity exhibited by HC neurons could be purely sensory in nature and independent of behavioral context. Several factors may account for the higher response selectivity observed in our study. First, the odor panels used were different. Second, behavioral significance and other task demands could alter odor selectivity of HC neurons. Third, our classification of odor response includes firing rate changes during and immediately after odor delivery and odor-induced respiratory tuning changes.

To the best of our knowledge, this is the first report of respiratory tuning of odor response in HC. This could underlie the synchronization between sniffing and theta rhythm during cue sampling and goal approach observed by Eichenbaum et al. (1987). It is interesting that respiratory tuning occurs so far downstream of the sensory input.

\section{Habituation}

Habituation to odors on prolonged or repeated stimulation is known to be a central phenomenon, distinct from receptor adaptation (Wilson, 1998). Mitral-tufted cells in OB show habituation with a slower onset and longer duration than receptor adaptation (Potter and Chorover 1976; Chaput and Panhuber, 1982). This effect is modulated by central inputs to OB, such that habituation occurs in response to nonreinforced odors but not in response to reinforced odors (Potter and Chorover, 1976; Grajski and Freeman, 1989; Wilson and Sullivan, 1992). Classically, odor habituation studies have concentrated on delivering prolonged 
odor stimulation. For studying habituation in $\mathrm{OB}$, stimuli were applied for $\sim 1 \mathrm{hr}$ (Chaput and Panhuber, 1982), whereas for studying habituation in piriform cortex and other higher-order processing areas, stimuli lasting 50-60 sec were used (Wilson, 1998; Poellinger et al., 2001). In contrast, our experiments consisted of $10 \mathrm{sec}$ odor delivery, repeated at different APs. This enabled us to perform statistical analyses over multiple trials. It also allowed us to examine the effect of habituation on neuronal responses to odor onset and turnoff events. Most importantly, this stimulation paradigm enabled us to examine habituation to intermittent stimuli, which might better mimic natural conditions such as distribution of odor in plumes (Vickers, 2000).

Our experiments with anesthetized, freely breathing rats show that, although most of the $\mathrm{OB}$ units respond to a given odor regardless of AP, odor responses of some of these were AP dependent in a manner suggestive of coarse tuning to AP. Local OB interactions and centrifugal inputs from higher-order brain regions might explain this phenomenon (Potter and Chorover, 1976). Experiments in awake animals have shown that odorresponsive properties of $\mathrm{OB}$ mitral-tufted cells are also modulated by behavioral context (Kay and Laurent, 1999), suggesting an additional role for the centrifugal inputs to $O B$.

Poellinger et al. (2001) performed functional magnetic resonance imaging recordings on human subjects while delivering odors for either 9 or $60 \mathrm{sec}$. HC responds to $9 \mathrm{sec}$ presentation with an increased BOLD signal. On prolonged, 60 sec exposure, primary olfactory cortex and $\mathrm{HC}$ showed an increased BOLD signal lasting for $10-15 \mathrm{sec}$, followed by a prolonged reduction in BOLD signal. This opens up the interesting possibility that HC forms a part of a central network involved in habituation and thus has a top-down influence on the process of odor perception.

We find that HC neurons show a marked AP selectivity in their odor responses. This observation supports the hypothesis of hippocampal involvement in habituation put forward by Poellinger et al. (2001) on the basis of functional magnetic resonance imaging studies. However, we do not see overall inhibition of HC neuronal activity on repeated presentations, as might be expected from their report. This might be caused by the difference in odor delivery paradigm. Alternatively, a reduction in the BOLD signal may not correspond directly to inhibition of pyramidal cell activity.

\section{Hippocampal function}

HC pyramidal cells have well documented place field selectivity, and this is known to be plastic depending on environment and task requirements (Redish, 2001). HC is also implicated in contextual memory and formation of memories representing relationships between different sensory stimuli. Redish (2001) postulates that there are multiple cognitive maps, spatial and nonspatial, within the HC and extrahippocampal regions of the brain and that the HC is involved primarily in "contextual recall." This would presumably necessitate the existence of highly processed sensory representations in the HC.

The AP selectivity and the absence of a discernible trend in HC single-unit responses toward longer or shorter APs was an unexpected result. If $\mathrm{HC}$ was not involved in odor habituation or representing the timing of the repeated odor stimuli, there should have been no selectivity for AP. If the $\mathrm{HC}$ was simply responding to the piriform cortex inputs, which show inhibition on prolonged odor presentation repeated at $30 \mathrm{sec}$ AP (Wilson, 1998), we should have seen more $\mathrm{HC}$ units preferentially responding to odors presented at longer APs than shorter APs. In contrast, we observed a fairly uniform distribution of selectivity of $\mathrm{HC}$ re- sponses to a range of APs. Thus, our results suggest that $\mathrm{HC}$ units are involved in encoding not just the odor stimuli but also the interval between repeated odor stimuli.

Approximately one-half of the $\mathrm{HC}$ units responding to more than one AP do so at nonadjacent APs. This was not because the responses were borderline significant, because $p$ values for adjacent APs, to which these neurons did not respond, were well above 0.05 . Furthermore, our analysis was sensitive enough to detect firing rate changes as low as $15 \%$. Coarse temporal coding, giving rise to responses to multiple adjacent APs, cannot explain the behavior of these neurons. More complex response patterns could occur with a sparse distributed code, with AP being encoded by an ensemble of neurons. One neuron can form a part of multiple ensembles, thus responding to multiple APs, which are not necessarily adjacent (Deadwyler and Hampson, 1997). Alternatively, some cells could encode higher-order features of the stimulus, such as complex cell responses in the visual system (Ohzawa et al., 1997). Consistent with either of these possibilities, we find that simultaneously recorded HC neurons include cells selective for single APs and cells selective for nonadjacent APs, showing that different response selectivities coexist.

Our analysis of trial-to-trial variability in odor response properties of the neurons suggests that simple changes in variability are not sufficient to account for AP selectivity. Characterization of the network interactions underlying AP selectivity will probably require simultaneous recordings of a large number of neurons from awake, behaving animals.

Our results indicate that $\mathrm{HC}$ could be involved in encoding odor identity and timing of repeated stimuli. This representation need not be completely independent of spatial and contextual learning, in which $\mathrm{HC}$ has been shown to be an important player. For example, the temporal coding described here could serve as a substrate for odor sequence memory (Agster et al., 2001; Fortin et al., 2002; Kesner et al., 2002). Natural odor stimuli are nonuniform, with a distinct temporal component. Crustaceans and insects have been shown to use the spatiotemporal aspects of the odor stimuli for tracking the source (Vickers, 2000). Our experiments show that $\mathrm{HC}$ neurons in rats respond to combinations of odor and time, suggesting that this may be a neuronal substrate for use of the temporal variation in odor stimuli for odor-guided navigation.

\section{References}

Agster FL, Fortin NJ, Eichenbaum H (2001) The hippocampus and disambiguation of overlapping sequences. J Neurosci 22:5760-5768.

Bhalla US, Bower JM (1997) Multiday recordings from olfactory bulb neurons in awake freely moving rats: spatially and temporally organized variability in odorant response properties. J Comput Neurosci 4:221-256.

Chaput M, Panhuber H (1982) Effects of long duration odor exposure on the unit activity of olfactory bulb cells in awake rabbits. Brain Res 250:41-52.

Chaput MA, Buonviso N, Berthommier F (1992) Temporal patterns in spontaneous and odor evoked mitral/tufted cell discharges recorded in anesthetized freely breathing animals. Eur J Neurosci 4:813-822.

Deadwyler SA, Hampson RE (1997) The significance of neural ensemble codes during behavior and cognition. Annu Rev Neurosci 20:217-244.

Eichenbaum H (2000) A cortical-hippocampal system for declarative memory. Nat Rev Neurosci 1:41-50.

Eichenbaum H, Kurperstein M, Fagan A, Nagode J (1987) Cue sampling and goal approach correlate of hippocampal unit activity in rats performing an odor discrimination task. J Neurosci 7:716-732.

Eichenbaum H, Otto TA, Wible CG, Piper JM (1991) Building a model of hippocampus in olfaction and memory. In: Olfaction: a model system for computational neuroscience (Davis JL, Eichenbaum H, eds), pp 167-210. Cambridge, MA: MIT. 
Fortin NJ, Agster KL, Eichenbaum H (2002) Critical role of the hippocampus in memory for sequences of events. Nat Neurosci 5:458-462.

Grajski KA, Freeman WJ (1989) Spatial EEG correlates of nonassociative and associative olfactory learning in rabbits. Behav Neurosci 103:790-804.

Habets AMMC, DaSilva FHL, Mollevanger WJ (1980) An olfactory input to the hippocampus of the cat: field potential analysis. Brain Res 182:47-64.

Hamilton KA, Kauer JS (1989) Patterns of intracellular potentials in salamander mitral/tufted cells in response to odor stimulation. J Neurophysiol 62:609-625.

Harrison TA, Scott JW (1986) Olfactory bulb responses to odor stimulation: analysis of response pattern and intensity relationships. J Neurophysiol 56:1571-1589.

Kay LM, Laurent G (1999) Odor- and context-dependent modulation of mitral cell activity in behaving rats. Nat Neurosci 2:1003-1009.

Kesner RP, Gilbert P, Barua LA (2002) The role of the hippocampus in memory for the temporal order of a sequence of odors. Behav Neurosci 116:286-290.

Lynch G, Granger R (1991) Serial steps in memory processing: possible clues from studies of plasticity in the olfactory-hippocampal circuit. In: Olfaction: a model system for computational neuroscience (Davis JL, Eichenbaum H, eds), pp 1141-1165. Cambridge, MA: MIT.

McNaughton BL, O’Keefe J, Barnes CA (1983) The stereotrode: a new technique for simultaneous isolation of several single units in the central nervous system from multiple unit records. J Neurosci Methods 8:391-397.

Ohzawa I, DeAngelis GC, Freeman R (1997) Encoding of binocular disparity by complex cells in the cat's visual cortex. J Neurophysiol 77:2879-2909.
Petersen RS, Panzeri S, Diamond ME (2001) Population coding of stimulus location in rat somatosensory cortex. Neuron 32:503-514.

Poellinger A, Thomas R, Lio P, Lee A, Makris N, Rosen BR, Kwong KK (2001) Activation and habituation in olfaction: an fMRI study. NeuroImage 13:547-560.

Potter H, Chorover SL (1976) Response plasticity in hamster olfactory bulb: peripheral and central processes. Brain Res 116:417-429.

Recce M, O’Keefe J (1989) An improved technique for multiunit extracellular recording. Soc Neurosci Abstr 15:1250.

Redish AD (2001) The hippocampal debate: are we asking the right questions? Behav Brain Res 127:81-98.

Vanderwolf CH (1992) Hippocampal activity, olfaction, and sniffing: an olfactory input to the dentate gyrus. Brain Res 593:197-208.

Vickers NJ (2000) Mechanisms of animal navigation in odor plumes. Biol Bull 198:203-212.

Wiebe SP, Staubli UV (1999) Dynamic filtering of recognition memory codes in the hippocampus. J Neurosci 19:10562-10574.

Wilson DA (1998) Habituation of odor responses in the rat anterior piriform cortex. J Neurophysiol 79:1425-1440.

Wilson DA, Sullivan RM (1992) Blockade of mitral/tufted cell habituation to odors by association with rewards: a preliminary note. Brain Res 594:143-145.

Wood ER, Dudchenko PA, Eichenbaum H (1999) The global record of memory in hippocampal neuronal activity. Nature 397:613-616.

Yokota T, Reeves AG, MacLean PD (1967) Intracellular olfactory response of hippocampal neurons in awake sitting squirrel monkeys. Science 157: 1072-1074. 